ORIGINAL BREVE

\title{
UTILIZACIÓN DE HORMONA DE CRECIMIENTO EN LA COMUNIDAD VALENCIANA DURANTE EL PERIODO 2003-2007
}

\author{
Ma Antonia Grau Rubio, Carmen Albelda Puig, Rosa Díaz Mondéjar y José Luís García Herrera.
}

Dirección General de Farmacia y Productos Sanitarios. Conselleria de Sanidad. Generalitat Valenciana.

\section{RESUMEN}

Fundamento: El objetivo de este estudio es analizar la evolución del consumo de hormona de crecimiento (Somatropina) en la Comunidad Valenciana, durante el periodo 2003 a 2007, determinando el impacto que ha tenido en el mismo el cambio de las condiciones de dispensación de diagnóstico hospitalario a uso hospitalario (mayo 2005), así como la aprobación de una nueva indicación en 2004. Se han estudiado también el número de pacientes tratados y el ahorro económico estimado por los tratamientos denegados por el Comité Asesor.

Métodos: Estudio descriptivo. Los datos sobre pacientes se han obtenido de la base de datos propia sobre protocolos de solicitud de tratamiento y los de consumo de hormona de crecimiento de las bases de datos de la Conselleria de Sanidad: Gestor de la prestación farmacéutica Gaia y de consumos de medicamentos en el hospital. Se expresan en número de pacientes tratados, miligramos dispensados y coste.

Resultados: El número de pacientes tratados durante este periodo ha pasado de 346 pacientes en 2003 a 520 en 2007 . Las denegaciones de tratamiento han supuesto un ahorro de 3.912.253 € a la Conselleria de Sanidad. El coste/mg de hormona de crecimiento ha pasado de $27,35 €$ en el 2003 , que se dispensaba a través de oficinas de farmacia, a $22,34 €$ en el 2007 que se dispensa desde los servicios de farmacia de hospital, lo que ha supuesto un ahorro del 18,3\% en el coste/mg considerando costes corrientes.

Conclusiones: El número de pacientes tratados aumentó un 50\% desde 2003 a 2007, habiendo influido la aprobación de una nueva indicación. El coste/mg ha disminuido un 18,3\% debido al cambio en las condiciones de dispensación. El Comité Asesor ha contribuido a que este medicamento se utilice según criterios de uso racional de medicamentos.

Palabras claves: Hormona de crecimiento. Utilización medicamentos. Gasto medicamentos.

Correspondencia:

$\mathrm{D}^{\mathrm{a}} \mathrm{M}^{\mathrm{a}}$ Antonia Grau Rubio

Servicio de Provisión y Asistencia Farmacéutica

Dirección General de Farmacia y Productos Sanitarios

Conselleria de Sanidad.

Calle Micer Mascó no 31 - 46010 - Valencia

Correo electrónico: grau_manrub@gva.es

\section{ABSTRACT}

\section{Utilization of Growth Hormone in Valencia Region, Spain, during the Period 2003-2007}

Background: The main goal is to analyze the evolution of the consumption of growth hormone (Somatropin) at the Valencia region (Spain). The research focuses on the period comprising 2003-2007 and aims at assessing the impact of the change in the conditions of dispensation of the hormone (May 2005 ) as well as of the approval of a new indication in 2004. We have also studied the number of treated patients and expenses saved in public money due to the denial of certain treatments.

Methods: The patient data have been obtained from a proprietary database owned by the region's government, this database contains information about the requests for treatment and the consumption of growth hormone has been obtained from database Gaia and drug consumption in the hospital. The data are expressed in terms of number of treated patients, consumed milligrams and cost.

Results: The number of patients treated with growth hormone has increased 50\% (from 346 patients in 2003 to 520 in 2007). The denial of requests for treatment has made it possible to save $3.912 .253 €$. The cost per $\mathrm{mg}$ of growth hormone has decreased from $27.35 €$ in 2003 , when the hormone was dispensed in local pharmacy stores, to $22.34 €$ in 2007 , when it was dispensed at hospital's pharmacies. The change in the conditions in which the drug is dispensed has led to a reduction of $18.3 \%$ considering current costs.

Conclusions: The number of treated patients has increased a 50\% from 2003 to 2007. In this period of time has been approved a new indication. The cost $/ \mathrm{mg}$ has been reduced a $18,3 \%$ due to the change in the conditions of dispensation. The Advisory Committee has contributed to the utilization of growth hormone according to rational drug use criteria.

Key words: Growth hormone. Drug utilization. Drug expenditure. 


\section{INTRODUCCIÓN}

La hormona de crecimiento (HC), también conocida como Somatropina, es una hormona producida en el lóbulo anterior de la hipófisis. Es esencial para el crecimiento normal en los niños y actúa directamente sobre las placas de crecimiento y a través de la producción de factores de crecimiento insulínico (especialmente el IGF-1) principalmente a nivel hepático. Tiene efectos también en el metabolismo de las proteínas, de los lípidos y de los carbohidratos, durante la infancia y en los adultos ${ }^{1,2}$.

En 1985, comenzó a producirse por ingeniería genética. Esta mayor disponibilidad de HC motivó la realización de ensayos clínicos y en consecuencia la ampliación de sus indicaciones. En nuestro país se autorizó como medicamento de Diagnóstico Hospitalario (DH) debido a sus posibles efectos adversos y requería el visado de inspección para su dispensación con cargo al Sistema Nacional de Salud. Pese a esta medida, se observó una utilización aparentemente superior a la de la previsible incidencia en la población. Por ello, se crearon Comités de Expertos en distintas Comunidades e INSALUD para supervisar la utilización de la $\mathrm{HC}^{3}$.

En la Comunidad Autónoma Valenciana se crea el Comité Asesor para la utilización terapéutica de la hormona de crecimiento y sustancias relacionadas, mediante Resolución de 16 de mayo de 1989 del Director del Servicio Valenciano de Salud (SVS). Dicho Comité actuaba como órgano consultivo del SVS, sobre el uso correcto de estos medicamentos, estableciéndose que para la dispensación de estos tratamientos con cargo al SVS deberían haber sido aprobados previamente por este Comité. Posteriormente, según se han ido autorizando nuevas indicaciones, se han actualizado los criterios de utilización así como los protoco$\operatorname{los}^{4,5}$.
En mayo del 2005, como consecuencia de una nota de seguridad de la Agencia Española del Medicamento, se modificaron las condiciones de dispensación de la $\mathrm{HC}$, pasando a calificarlo como medicamento de uso hospitalario (UH). A partir de este momento su dispensación se realizará desde los Servicios de Farmacia de Hospital ${ }^{6}$.

En la actualidad las indicaciones aprobadas son:

\section{En niños:}

1. Trastorno del crecimiento por secreción insuficiente de hormona de crecimiento (deficiencia de hormona de crecimiento)

2. Trastorno de crecimiento en niñas con Síndrome de Turner ${ }^{7}$.

3. Trastorno del crecimiento en niños prepuberales con insuficiencia renal crónica.

4. En el Síndrome de Prader-Willi, para mejorar el crecimiento y la composición corporal.

5. Trastorno de crecimiento en niños con talla baja nacidos pequeños para su edad gestacional (PEG), última indicación aprobada.

\section{En adultos:}

6. Terapia sustitutiva en adultos con un déficit marcado de hormona de crecimiento.

La HC recombinante, está comercializada en España con varios nombres registrados y presentaciones. Cada $\mathrm{HC}$ es producida por ingeniería genética y tiene una secuencia idéntica a la HC humana. Las indicaciones aprobadas para estos medicamentos son ${ }^{8}$ : 
Genotonorm-R.- todas las indicaciones arriba referenciadas (1-6)

Humatrope-R.- indicaciones 1, 2, 3, 4 y 6 además tiene aprobada la indicación:

Tratamiento de pacientes con deficiencia de crecimiento asociado a alteraciones del gen SHOX, confirmada mediante análisis genético.

Norditropin-R.- indicaciones 1, 2, 3, 4 y 6

Saizen-R.- indicaciones $1,2,3,4$ y 6

Zomacton-R.- indicaciones 1, 2

Omnitrope-R.- indicaciones 1-6 (primer medicamento biosimilar autorizado en nuestro país)

Las dosis se establecen en base al peso del paciente y varían según la indicación aprobada, las recomendadas son: 0,025$0,035 \mathrm{mg} / \mathrm{Kg} /$ día para déficit de $\mathrm{HC}, 0,045$ 0,050 mg/kg/día para Síndrome de Turner y para Insuficiencia Renal Crónica, 0,035 $\mathrm{mg} / \mathrm{kg} /$ día para Síndrome de Prader Willi y para niños con talla baja nacidos pequeños para su edad gestacional ${ }^{7}$. La dosis inicial para adultos es $0,2-0,3 \mathrm{mg}$ (generalmente $0,27 \mathrm{mg}$ ) y la dosis de mantenimiento más usual es de $0,4 \mathrm{mg} .^{2}$.

El objetivo del presente trabajo es analizar la evolución del número de pacientes tratados con $\mathrm{HC}$ recombinante, para cada una de las indicaciones aprobadas, durante el periodo 2003-2007, en el que cambian las condiciones de dispensación, se aprueba una nueva indicación para alguno de estos medicamentos y se comercializa el primer medicamento biosimilar en nuestro país.

\section{MATERIAL Y MÉTODOS:}

Estudio descriptivo del número de sujetos tratados con $\mathrm{HC}$ en la Comunidad
Valenciana, desde 2003 hasta 2007, obtenidos de la base de datos propia de la Conselleria de Sanidad sobre protocolos evaluados por el Comité Asesor para las distintas indicaciones autorizadas. Se han relacionado estos datos con los datos de consumo y costes económicos obtenidos del sistema gestor de la prestación farmacéutica Gaia, que recoge los medicamentos dispensados con receta oficial en oficinas de farmacia, con cargo a la Conselleria de Sanidad (2003-mayo 2005) periodo en el que estos medicamentos estaban calificados como DH. Desde junio de 2005 a diciembre de 2007, los datos de consumo y costes económicos hospitalarios, se han obtenido de la base de datos propia de consumos de medicamentos en los hospitales en la Conselleria de Sanidad. Las cifras de población se han obtenido del Instituto Nacional de Estadística ${ }^{9}$, en el padrón municipal se han consultado los datos anuales de población de la Comunidad Valenciana correspondientes al período de estudio.

Los datos se expresan como: número de pacientes tratados por indicación, coste/mg que se ha calculado a partir del coste de estos medicamentos dispensados con cargo a la Conselleria de Sanidad y los mg totales dispensados, coste/paciente/año que se ha calculado a partir del coste total año y el número de pacientes tratados. El ahorro estimado de los protocolos denegados por el Comité Asesor se ha calculado a partir del importe medio anual del tratamiento por paciente, asumiendo una duración media de 5 años para los tratamientos de inicio y de 2,5 años para tratamientos de seguimiento.

\section{RESULTADOS}

El número de pacientes tratados durante este periodo ha aumentado un $50 \%$, de 346 pacientes en 2003 a 520 en 2007. Este incremento es debido sobre todo al aumento de niños tratados con déficit de HC $(58 \%)$ y a los nuevos tratamientos 


\section{Figura 1}

Pacientes tratados con HC con cargo a la Conselleria de Sanitat en la Comunidad Valenciana durante el período 2003-2007

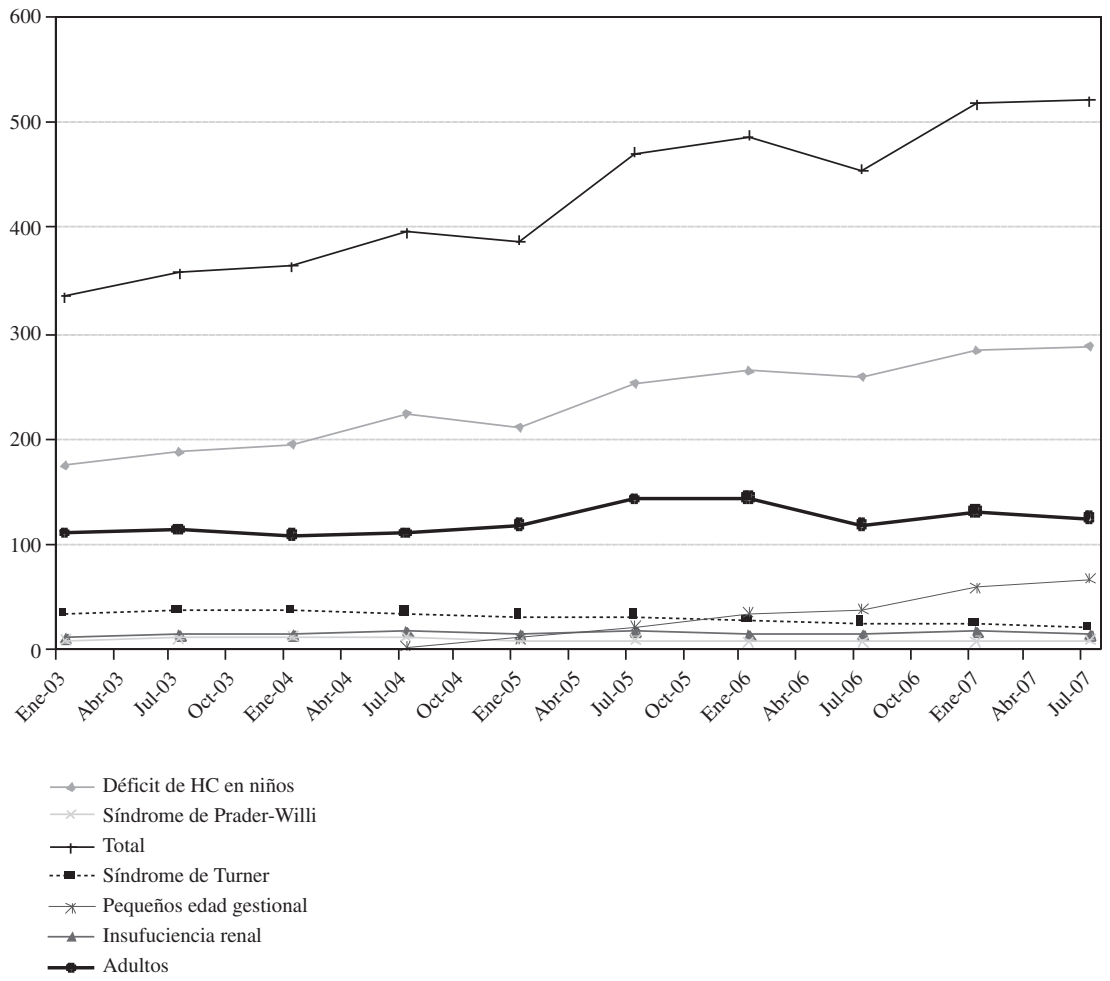

para PEG, a finales del 2007 ya se estaban tratando 66 niños con esta patología (figura 1).

El ahorro estimado de los protocolos denegados por el Comité Asesor para estos 5 años asciende a 3.912.253 $€$ y se ha cal- culado a partir del coste medio anual del tratamiento por paciente (tabla 1$)^{3}$.

En la tabla 2 se recogen semestralmente los datos correspondientes a: número de pacientes tratados, mg totales dispensados, importe de los medicamentos dispensados a

Tabla 1

Estimación del ahorro en la prescripción de HC en la Comunidad Valenciana con cargo a la Conselleria de Sanidad durante el período 2003-2007 (en euros)

\begin{tabular}{|l|r|r|r|r|r|}
\hline & $\mathbf{2 0 0 3}$ & $\mathbf{2 0 0 4}$ & $\mathbf{2 0 0 5}$ & $\mathbf{2 0 0 6}$ & $\mathbf{2 0 0 7}$ \\
\hline Coste anual paciente & 8.565 & 9.184 & 8.741 & 9.289 & 7.709 \\
\hline Tratamientos inicio denegados & 13 & 33 & 27 & 30 & 54 \\
\hline Tratamientos seguimiento denegados & 1 & 7 & 12 & 7 & 5 \\
\hline Ahorro total* & 587.464 & 1.310 .446 & 957.567 & 601.945 & 454.831 \\
\hline
\end{tabular}

* Se ha estimado que los tratamientos de inicio denegados tendrían una duración media de 5 años y los de seguimiento 2'5. 
Tabla 2

Tratamiento con HC en la Comunidad Valenciana con cargo a la Conselleria de Sanidad durante el período 2003-2007

\begin{tabular}{|c|c|c|c|c|c|c|c|c|c|c|}
\hline & ene-jun 03 & jul-dic 03 & ene-jun 04 & jul-dic 04 & ene-jun 05 & jul-dic 05 & ene-jun 06 & jul-dic 06 & ene-jun 07 & jul-dic 07 \\
\hline $\mathrm{n}^{\circ}$ pacientes & 335 & 357 & 364 & 395 & 387 & 471 & 488 & 456 & 518 & 521 \\
\hline $\mathrm{mg}$ totales* & 51.421 & 56.928 & 62.919 & 65.315 & 82.499 & 72.794 & 102.837 & 92.587 & 91.100 & 88.326 \\
\hline importe** & 1.406 .367 & 1.557 .274 & 1.709 .876 & 1.779 .991 & 2.099 .777 & 1.649 .914 & 2.316 .307 & 2.067 .878 & 2.029 .813 & 1.978 .946 \\
\hline coste/mg & $27 ’ 35$ & $27 ' 36$ & $27^{\prime} 18$ & $27 ' 25$ & $25{ }^{\prime} 45$ & $22{ }^{\prime} 67$ & $222^{\prime} 52$ & $22^{\prime} 33$ & $222^{\prime} 28$ & $22^{\prime} 40$ \\
\hline coste/paciente/semestre & 4.198 & 4.362 & 4.697 & 4.506 & 5.426 & 3.503 & 4.747 & 4.535 & 3.919 & 3.798 \\
\hline coste/paciente/día & $23^{\prime} 00$ & $23^{\prime} 90$ & $25 ’ 74$ & $24^{\prime} 69$ & $29^{\prime} 73$ & $199^{\prime} 19$ & $26^{\prime} 01$ & $24^{\prime} 8$ & $21^{\prime} 47$ & $20 ' 81$ \\
\hline $\mathrm{mg} /$ paciente/día & 0'84 & 0,87 & $0 ’ 95$ & 0'92 & 1'17 & 0,85 & 1'15 & 1'11 & $1 ’ 09$ & $0 ’ 93$ \\
\hline
\end{tabular}

* Se calculan a partir de los mg que contiene cada envase multiplicado por los envases dispensados.

** Corresponde al coste de los medicamentos dispensados desde oficina farmacia (hasta abril 2005) y desde servicios farmacia hospital (mayo 05-diciembre 07).

Tabla 3

Prescripción de HC en la Comunidad Valenciana con cargo a la Conselleria de Sanidad durante el período 2003-2007

\begin{tabular}{|c|c|c|c|c|c|c|c|c|c|c|}
\hline & ene-jun 03 & jul-dic 03 & ene-jun 04 & jul-dic 04 & ene-jun 05 & jul-dic 05 & ene-jun 06 & jul-dic 06 & ene-jun 07 & jul-dic 07 \\
\hline mg totales & 51.421 & 56.928 & 62.919 & 65.315 & 82.499 & 72.794 & 102.837 & 92.587 & 91.100 & 88.326 \\
\hline $\mathrm{DDD}^{*}$ & 77.132 & 85.392 & 94.379 & 97.973 & 123.749 & 109.191 & 154.256 & 138.881 & 136.650 & 132.489 \\
\hline DHD $^{* * *}$ & 0,095 & 0,105 & 0,114 & 0,118 & 0,145 & 0,128 & 0,176 & 0,158 & 0,153 & 0,149 \\
\hline
\end{tabular}

* DDD (Dosis Diaria Definida) es de 2UI (1mg=3UI), establecida por WHO Collaborating Centre for Drug Statistics Methodology (2008).

** DHD (DDD por 1000 habitantes y día).

través de oficina de farmacia y de servicios de farmacia de hospital, el coste/mg, el coste por paciente y semestre, $\mathrm{y} \mathrm{mg}$ por paciente y día. Cabe destacar el aumento de dosis dispensadas, debido al mayor número de pacientes tratados, y consecuentemente el incremento del coste total de los tratamientos con HC. Por otro lado el coste por mg disminuye al dispensarse la $\mathrm{HC}$ desde los servicios de farmacia de hospital, ya que en el precio no se incluye los márgenes de la distribución ni de las oficinas de farmacia.

Aunque no se han utilizado datos de DHD (Dosis Diaria Definida por 1000 habitantes y día) porque se disponía del número de pacientes tratados, se incluye una tabla con las DHD con el fin de facilitar la comparación con otros trabajos que utilicen esta unidad de medida (tabla 3$)^{10,11}$.

\section{DISCUSIÓN}

Para la interpretación de los datos del presente estudio se debe tener en cuenta algunas limitaciones:
- Las fuentes de información utilizadas no contemplan el consumo que se produce a cargo de otras entidades gestoras como MUFACE, ISFAS, entre otras.

- Aunque realmente se utilizan términos como uso y consumo, lo que realmente se mide es dispensación, que equivaldría al consumo solo cuando el cumplimiento fuese perfecto.

A diferencia de otros trabajos ${ }^{3,12}$ que utilizan datos de coste por paciente y patología estimados en base a las dosis recomendadas y el precio de comercialización de los medicamentos, en este trabajo se recogen los datos de coste real por paciente calculados a partir del importe de los medicamentos dispensados con cargo a la Administración Sanitaria Valenciana y del número de tratamientos.

Aunque no se han utilizado datos de DHD, se incluyen en la tabla 3 con el fin de facilitar la comparación con otros trabajos que utilicen esta unidad de medida ${ }^{10,11}$.No se ha expresado el consumo en DHD porque: 
- Conocemos el número de pacientes tratados por indicación y la DDD (dosis diaria definida) es una unidad técnica de medida que permite establecer comparaciones pero que no necesariamente coincide con la dosis utilizada por la población.

- Los datos que se han manejado han sido expresados en mg, dado que es la unidad de dosificación para la $\mathrm{HC}$ (mg/Kg/día) y ésta varía según la indicación ,sin embargo la DDD viene en unidades internacionales (UI) ${ }^{13}$. La DDD es de 2 UI y $1 \mathrm{mg}$ de $\mathrm{HC}$ equivale a $3 \mathrm{UI}$.

Para explicar la evolución del consumo de HC y su coste debemos considerar diversos factores como la aprobación de una nueva indicación, el paso a dispensación hospitalaria, la comercialización de un medicamento biosimilar y la existencia del Comité Asesor.

La nueva indicación para niños nacidos con bajo peso para su edad gestacional, que se hace efectiva a partir de enero de 2005 contribuye al aumento de los pacientes tratados junto con el aumento de los niños tratados con déficit de HC.

En cuanto al coste/mg, se mantiene prácticamente constante alrededor de $27 € / m g$ hasta el punto de inflexión que supuso el paso a uso hospitalario en mayo del 2005, disminuyendo en el primer semestre de 2005, donde coexisten los dos sistemas de dispensación y que pasa a un valor próximo a $25 € / \mathrm{mg}$, que de nuevo experimenta una disminución a partir del segundo semestre de 2005 aproximándose a $22 €$. Esto se explica porque el coste de los medicamentos dispensados a través de servicios de farmacia de hospital no incluye los márgenes de la distribución ni de la oficina de farmacia.

En el 2007 empieza a consumirse el medicamento biosimilar, que aunque solo representa un $0,72 \%$ del consumo total de
$\mathrm{HC}$ en $\mathrm{mg}$, el coste/mg de este medicamento $(18,88 €)$ es un $15,6 \%$ inferior al coste medio por mg de las otras marcas comerciales con HC dispensadas con cargo a la Conselleria de Sanidad en este año.

El funcionamiento del Comité Asesor ha tenido repercusión tanto en lo que se refiere al gasto farmacéutico como a la introducción de criterios diagnósticos y terapéuticos para la utilización de HC. Así se ha obtenido un ahorro real de 3.912.253€ correspondiente a los protocolos denegados durante el periodo de estudio $\mathrm{y}$, por otra parte ha aumentado el conocimiento y la aplicación de los criterios para la utilización de $\mathrm{HC}$, lo que ha hecho que un elevado porcentaje de los tratamientos evaluados por este Comité Asesor se haya informado favorablemente.

\section{AGRADECIMIENTOS}

Se agradece la labor de todos los profesionales que han pertenecido al Comité Asesor.

\section{BIBLIOGRAFÍA}

1. National Institute for Clinical Excellence. Guidance the use of human growth hormone (somatropin) children with growth failure. Technology Appraisal Guidance- No. 42. Mayo 2002. Disponible en: www.nice.org.uk

2. National Institute for Clinical Excellence. Human growth hormone (somatropin) in adults with growth hormone deficiency. Technology Appraisal Guidance- No 64. Agosto 2003. Disponible en: www.nice.org.uk

3. García Comas L, Conde Olasagasti JL. Uso terapéutico de la hormona de crecimiento en el INSALUD. Med Clin. 1998; 111: 542-545.

4. Resolución de 16 de Mayo de 1989 del Director del Servicio Valenciano de Salud por la que se crea el comité asesor para la utilización terapéutica de la hormona de crecimiento y sustancias relacionadas.

5. Circular 23/89 del Director Servicio Valenciano de Salud (SVS) de 22 de noviembre de 1989 
(modificada por resolución de SVS de 8 de noviembre de 1994).

6. Riesgos de uso de la hormona de crecimiento en jóvenes y adultos sanos, y paso a Uso Hospitalario. Nota informativa 2005/08 de la AEMPS. Disponible en: www.agemed.es/actividad/alertas/usoHumano/seguridad/Hcrecimiento.htm

7. Baxter L, Bryant J, Cave CB, Milne R. Hormona de crecimiento recombinante en niñas y adolescentes con síndrome de Turner (Revisión Cochrane traducida). En: La Biblioteca Cochrane Plus, 2007 Número 4. Oxford: Update Software Ltd. Disponible en: http://www.update-sofware.com. (Traducida de The Cochrane Library, 2007 Issue 4. Chichester, UK: John Wiley \& Sons, Ltd.).

8. Fichas técnicas. Agencia Española del Medicamento y Productos Sanitarios. Disponible en: htpps://sinaem4.agemed.es/consaem/fichasTecnicas.do?metodo $=$ detalleForm
9. Instituto Nacional de Estadística. Disponible en: www.ine.es/inebmenu/mnu_cifraspob.htm

10. García-Iñesta A. Estudios de utilización de medicamentos en España y análisis de la situación farmacoterapéutica. En: Instituto Nacional de la Salud, editor. Estudios de utilización de Medicamentos. Madrid: Instituto Nacional de la Salud, 1992; 15-48.

11. Martín-Arias LH, Carvajal A. El consumo de medicamentos en España. Valladolid: Universidad de Valladolid, 1994.

12. Vaquero García MD, Yécora Navarro MG. Estudio utilización de hormona de crecimiento 2002. Inf Ter Sist Nac Salud. 2003; 27: 76-79.

13. WHO Collaborating Centre for Drug Statistics Methodology. Disponible en: www.whocc.no/atcddd. 
